\title{
Applying Cervical Spine Anatomy to Interscalene Brachial Plexus Blocks
}

\author{
Edward Carden, MD, and Arti Ori, MBChB
}

Background: The interscalene brachial plexus block (ISBPB) is a most reliable and commonly performed technique for regional anesthesia of the upper extremity. It has widespread clinical applicability, ranging from use for shoulder surgery as well as diagnostic and therapeutic uses in pain management. Traditional methods described for performing the ISBPB involve identifying surface anatomy landmarks. Unfortunately, in patients with less than ideal landmarks (those with short, thick necks and those lacking adequate muscle tone in the neck area) it be-

The interscalene brachial plexus block (ISBPB) has become a most reliable and commonly performed technique for regional anesthesia of the upper extremity. It has widespread clinical applicability including use for shoulder surgery, as well as pain management (1-6). In addition to the provision of post-operative analgesia, ISBPB is frequently used by interventional pain physicians for the treatment of chronic upper extremity pain.

The brachial plexus is formed by the union of the anterior rami of cervical fifth, sixth, seventh, eighth, and first thoracic nerves, with contributions from the fourth cervical and second thoracic

From: Department of Anesthesiology, University of Southern California, Keck School of Medicine, Los Angeles, California; and Southern California Academic Pain Management, Marina Del Rey, California

Address correspondence:

Edward Carden, MD

c/o DISC

1316o Mindanao Way, Suite 300

Marina Del Rey, CA 90292

E-mail: tedcarden@comcast.net

Disclaimer: There was no external funding in the preparation of this manuscript

Conflict of Interest: None

Manuscript received on 5/15/2005

Revision submitted on 9/1/2005

Accepted for publication on 9/03/2005 comes increasingly challenging to identify these landmarks. As a result there is greater uncertainty in accurately locating the brachial plexus, and consequently greater risk in performing the block.

Methods: A simple new approach to the interscalene brachial plexus block is described, utilizing the bony anatomy of the cervical spine as a landmark for directing the needle to the correct position, a nerve stimulator, and a confirmatory injection of a test dose of anesthetic solution to enhance accuracy. In addition, by correctly implementing this technique, the block may be performed by a sole operator.

Results: This simple approach has proven to be clinically effective in more than 2,000 blocks of the brachial plexus during the past 4 years.

Conclusion: It is concluded that this technique represents a safe, reproducible, and highly successful method for use by anesthesiologists and pain physicians alike.

Keywords: Interscalene block, upper extremity pain, regional anesthesia for upper extremity nerves. It is believed that these nerves are enclosed in a continuous fascial sheath, as highlighted by Winnie (7). Thus, if one is able to insert a needle inside the sheath, then all the nerves of this plexus can be blocked. This existence of a brachial plexus sheath allows for effective blockade of the brachial plexus, with techniques that are performed above the clavicle. The interscalene block is performed high in the plexus at the level of the roots and trunks. Contributing to its popularity is the technical simplicity and inherent safety that it provides, as the ISBPB is performed well superior to the subclavian artery and dome of the pleura.

However, there exists potential for complications if the person performing the block is not completely familiar with the technique, or with the relevant anatomy involved. Using the traditional methods that have been described, the ISBPB is carried out by identifying surface anatomy landmarks. The operator palpates the posterior border of the sternocleidomastoid muscle until the interscalene groove is first identified and then marked at the level of the cricoid cartilage (8). Unfortunately, in patients with short, thick necks and those that lack adequate muscle tone in this area, it becomes increasingly challenging to identify these visual landmarks.
The result is that the block becomes more perilous to perform, with greater risk for complications and failure. Our technique for the ISBPB was developed to overcome these limitations and provide a safe, consistent technique. This short communication will describe a new and simple approach using the bony anatomy of the cervical spine, which has proven to be effective in 2,000 consecutive injections of the brachial plexus.

\section{Applied Anatomy}

This approach utilizes the bony anatomy of the cervical spine as a method for directing the needle to the correct position. The brachial plexus is formed by the union of the anterior rami of the cervical fifth, sixth, seventh, eighth, and first thoracic nerves, with contributions from the fourth cervical and second thoracic nerves. In order to ensure that one has located these nerves correctly a nerve stimulator is used, while to preliminarily locate the position of the plexus, the bony anatomy is used.

The nerves of the brachial plexus exit through the intervertebral foraminae, and run laterally in a deep groove or gutter on the superior surface of the transverse process of each cervical vertebra (9). Each transverse process ends in an ante- 
rior tubercle and a posterior tubercle, between which the spinal nerve passes, running downward in the neck toward the first rib. The direction of the gutters is laterally, slightly anterior, and almost 45 degrees caudad. The prominent anterior tubercle of the C6 transverse process, which protrudes laterally, can easily be felt by the searching fingers. The nerve plexus can be located by placing one finger in front of this tubercle, and thus in front of the transverse process, and one finger behind. The prominent anterior tubercle lies between the two fingers, slightly closer to the anterior finger. Once it is located, a shielded needle can be directed medially and caudally to identify and enter the brachial plexus sheath (Fig. 1). Directing the needle caudally ensures that the needle does not inadvertently enter the spinal canal.

\section{Materials and Methods}

Peripheral nerve stimulator (10)

22-gauge, B-bevel shielded needle (11)

Grounding pad or EKG pad

20 cc syringe

Extension tubing (short or long)

Local anesthetic agent of choice

For skin infiltration: 30-gauge needle with syringe and local anesthetic solution

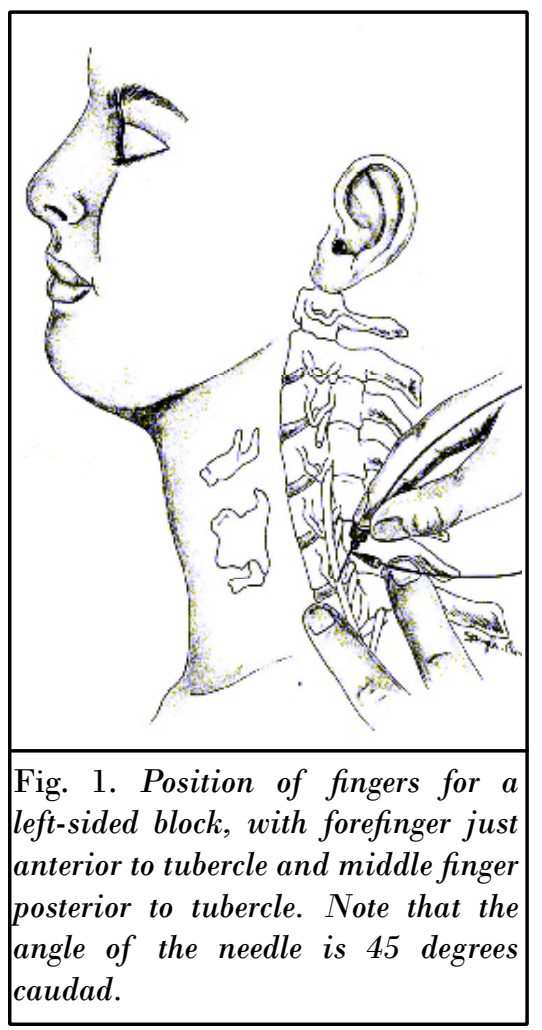

1. It is preferable to have the patient connected to an EKG monitor. The patient is positioned supine on a gurney, with the head turned away from the side to be blocked. The neck is aseptically prepared, and the operator performing the block is wearing sterile gloves. In a righthanded person, the left hand is the palpating hand. The prominent anterior tubercle of the C6 transverse process is palpated at the level of the cricoid cartilage (C6/7). For a rightsided block, the forefinger is placed finger is placed behind the tubercle, and significant pressure is directed medially. The opposite is done for a left-sided block.

2. With the lateral soft tissue of the neck compressed against the spine, the fingers are now in front of and behind the transverse process. The nerve plexus lies between the two fingers, slightly closer to the anterior finger. Anesthesia of the subcutaneous tissues at the projected site of needle entry is obtained by infiltrating $0.5 \mathrm{ml}$ of $1 \%$ plain lidocaine into the skin using a 30 -gauge needle. Afterwards, the 22-gauge shielded Bbevel needle, which is attached to the in front of this tubercle, the middle

nerve stimulator (Fig. 2), is inserted at a point slightly anterior from the mid-point between the forefinger and middle finger, directed 45 degrees in a caudal direction. As mentioned earlier, this is done at the level of the cricoid cartilage. It is critically important to keep the needle in a 45degree angle directed caudad; failure to do so might allow the needle to pass into the intervertebral foramen (Fig. 3).

3. The use of a nerve stimulator is not only a great advantage, but is necessary for the proper location of the brachial plexus, particularly in patients who are inebriated, sedated, or obtunded from their trauma. Utilizing $2 \mathrm{~Hz}$ delivered by the nerve stimulator, the needle is advanced slowly until stimulation resulting in motion from the shoulder is obtained (12). If the tubercle is contacted prior to nerve identification, the needle should be withdrawn to the skin and redirected to the proper course.

4. On obtaining appropriate stimulation at a suitably low current level (e.g., $<0.5 \mathrm{~mA}$ ), gentle aspiration is performed, followed by injection of a test dose of $1 \mathrm{ml}$ of the local anesthetic agent. At this point all stimulation

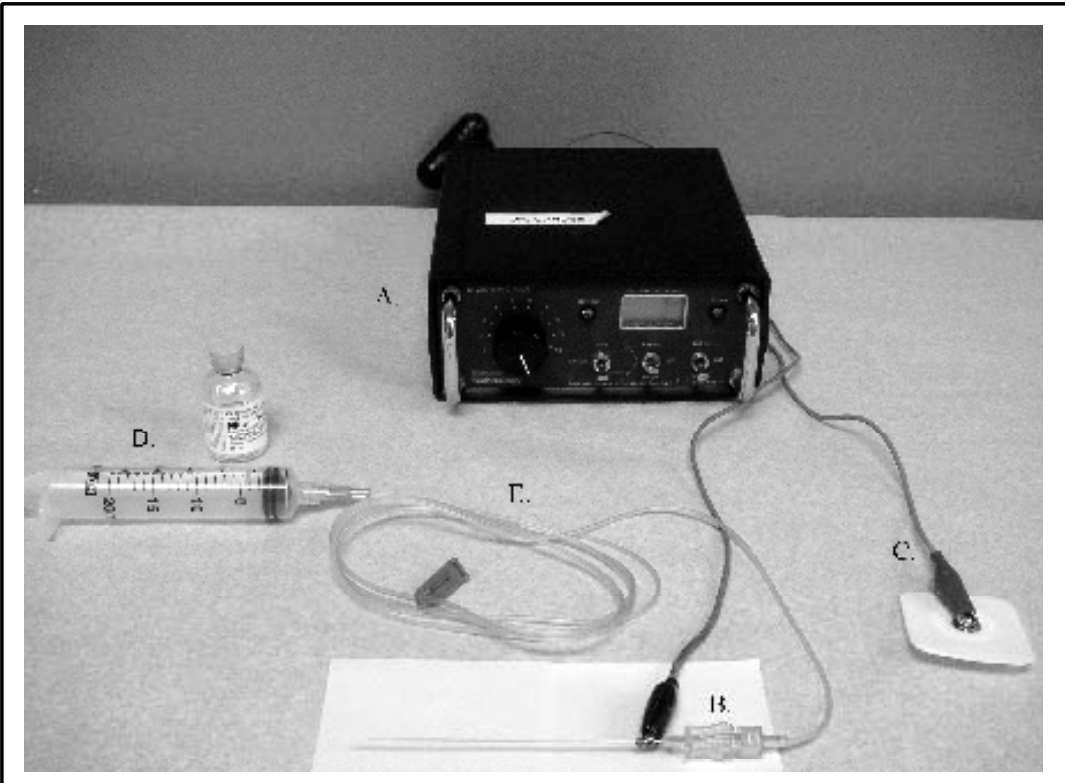

Fig. 2. (A) Peripheral nerve stimulator; (B) 22-gauge B-bevel shielded needle with lead attached by alligator clip to the negative electrode; (C) lead connected to EKG pad; (D) $20 \mathrm{ml}$ syringe with local anesthetic agent; and (E) extension tubing. 


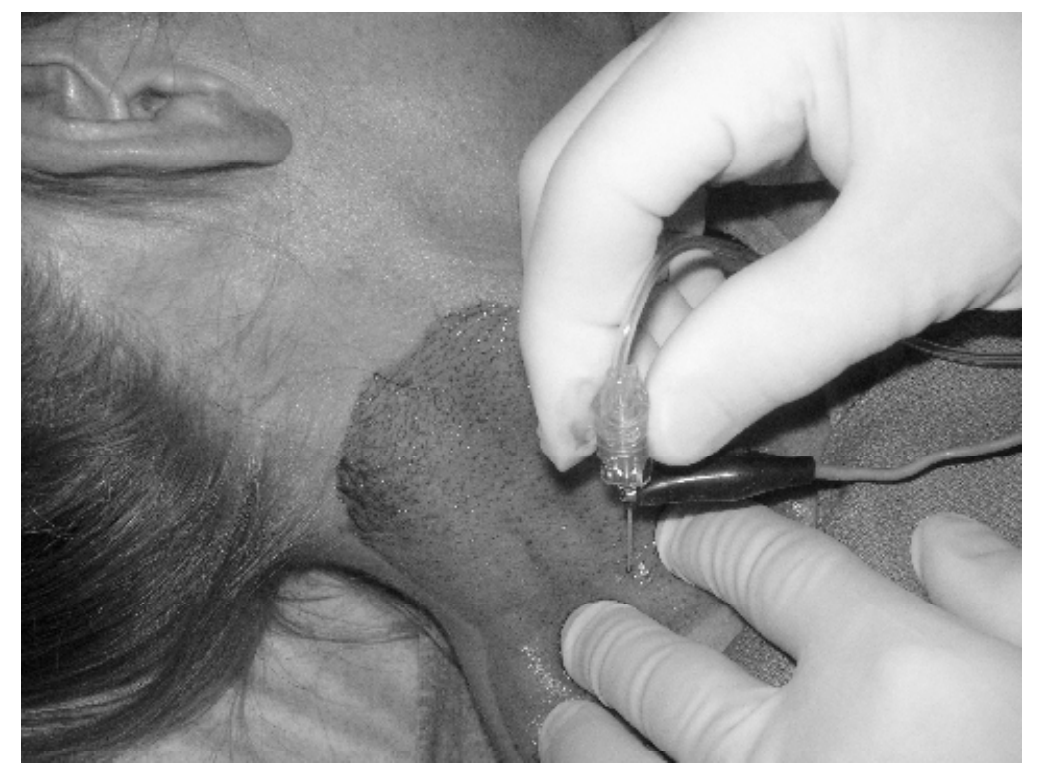

Fig. 3. Right-sided interscalene block

should cease. If all stimulation ceases, $15-20 \mathrm{ml}$ of anesthetic solution is injected incrementally, with aspiration being performed at every 3-5 ml increment. Up to $40 \mathrm{ml}$ can be used, although sufficient block is often obtained with smaller volumes. While the injection is carried out, the pressure exerted by the forefinger and middle finger are to be maintained firmly against the cervical spine. This will stabilize the needle, holding it in its proper position. Throughout the entire procedure, the palpating hand should not be moved. Observation of the patient is carefully conducted, paying particular attention to signs of possible intravascular injection. It is always best to perform this block with a local anesthetic containing epinephrine so that while the injection is being carried out, a tachycardia will develop should the needle penetrate a blood vessel $(13,14)$.

5. If stimulation does not cease, then the tip of the needle is not in the sheath and the needle will need to be repositioned. In this instance the needle could be right next to the sheath and be stimulating the nerves, but not actually be inside the sheath which is the target site for the local anesthetic. The needle should be adjusted slightly laterally and medially to produce maximum stimula- tion with the nerve stimulator. Once the needle is deemed to be correctly positioned, and provided that good pressure is exerted down on the tissues (which will hold the needle solidly in position), the rest of the medication can be injected with a high certainty of success.

6. One can expect the block to set up in approximately 10-15 minutes. The success rate of doing the block in this manner is close to $100 \%$. The patient is monitored for the extent of anesthesia of the upper extremity and surgical preparation is begun, if necessary. A Horner syndrome may develop; however, more attention is required for monitoring possible intravascular injection, or shortness of breath that may indicate possible pneumothorax (9,15-17).

\section{Complications}

There is a $3 \%-30 \%$ chance of a missed nerve block with the traditional approach of the ISBPB $(16,18)$. With the technique described here, granted that pressure is maintained firmly against the cervical spine throughout the injection period, a nerve stimulator is used, and all stimulation ceases with the injection of a $1 \mathrm{ml}$ test dose of solution, there is an expected minimal occurrence of a failed block.

In general, pneumothorax rarely oc- curs. The pleura may be punctured if the needle is directed too far inferiorly. Transient neuropathy and neuropathy with permanent dysfunction are also rare, particularly with this technique, as paraesthesiaes are not sought (19).Unintentional epidural and spinal blockade has been reported as a complication of interscalene block $(20,21)$. However provided the needle is directed in a 45 degree caudal direction, the risk of entering the intervertebral space or injection into the dural sleeves is minimized. An interscalene block inevitably results in ipsilateral diaphragmatic paralysis due to phrenic nerve blockade (22). However the majority of patients have enough pulmonary reserve that this is not significant with unilateral blocks. Blockade of the recurrent laryngeal nerve with resultant hoarseness, as well as blockade of the cervical sympathetic nerves resulting in a transient Horner syndrome may occur; these are not of major clinical significance. The most serious potential complication is intravascular injection. This may lead to seizures if injection occurs under high pressure and reverse flow occurs. It also is necessary to evaluate the patient's blood coagulation status before performing the procedure. All the above complications may be minimized with the correct implementation of this technique.

\section{DISCUSSION}

With the publication of his classic paper in 1970 (1), Winnie popularized the interscalene block as a technique for regional anesthesia of the upper extremity. He suggested this technique for prolonged surgeries of the upper extremities. Manriquez and Pallares (2), who described the use of the ISBPB for post-operative pain management, supplemented Winnie's work. Kirkpatrick et al (3) further developed the application of the ISB$\mathrm{PB}$ in 1985, demonstrating the long-term usefulness of this technique when administering constant infusions to patients for as long as 4 days. From its use as a regional anesthesia technique for surgical procedures in the operating room, to a useful diagnostic and therapeutic technique for interventional pain management in a physician's office, the widespread clinical applicability of the ISBPB has resulted in it being a very popular and commonly performed technique. The technical simplicity and inherent safety that it provides contributes to the appeal of this tech- 
nique. The ISBPB is performed well superior to the subclavian artery and cupula of the pleura.

The main determining factor for success with any regional block is technical skill; there exist situations that rigorously challenge this skill and increase the risk for complications. Using the traditional methods that have been described, the ISBPB is carried out by identifying surface anatomy landmarks. However some patients have less-than-ideal landmarks: those with short, thick necks, and those that lack adequate muscle tone in the neck area. In these cases, the visual landmarks become increasingly challenging to identify, with the result that the block becomes more hazardous to perform. Thus, there exists a greater risk for complications and failure of the block.

The technique described here for the ISBPB overcomes the limitations of relying on surface anatomy landmarks. By applying the anatomy of the cervical spine in locating the easily palpable osseous landmarks, the position of the brachial plexus is accurately and consistently located. Although others have previously described similar methods of locating the brachial plexus at the level of the roots (23), to increase accuracy, and in order to confirm that one has located these nerves correctly, a nerve stimulator is employed in this technique, as well as a test dose of $1 \mathrm{ml}$ of anesthetic solution to confirm the position of the needle in the sheath. In addition, with significant pressure applied against the cervical spine, the tissues become stiff and the needle is held firmly in place. One can then utilize the other hand to inject the solution. This allows for performance of the block by a solo operator as has been done at the authors' institution.

It is important to note that the needle is directed at approximately 45 degrees in a caudal direction in order to ensure that the needle does not enter the spinal column itself, and that the vertebral artery is not inadvertently injured. Injection of the initial test dose will immediately create significant problems for a patient if either of these two incidences has occurred. One should also ensure that the patient is fully monitored. It is always best to do this block with local anesthetic containing epinephrine so that while the injection is being carried out, a tachycardia will develop if the needle penetrates a blood vessel. Finally, if the needle is in a blood vessel, the injection of $1 \mathrm{ml}$ of local anesthetic will not ablate stimulation.

At the authors' practice, this technique has been performed on all brachial plexus block cases from 1990 to 2004 . Approximately 2,000 consecutive injections during the past 48 months have been carried out with a very high success rate $(>95 \%)$. There were no incidences of intra-arterial injection, pneumothorax, inadvertent subarachnoid puncture, or toxic effects of the local anesthetic. With this technique the authors do not expect a Horner syndrome to result, as the block is performed at the C6/7 level and the superior cervical ganglion is not blocked.

\section{CONCLUSION}

The ISBPB is a popular and commonly performed technique for regional anesthesia of the upper extremity for surgical procedures, and also as a useful diagnostic and therapeutic technique for pain management. Traditional methods of performing the ISBPB using surface anatomy have fallen short in patients where it is difficult to identify the proper surface landmarks. We have described a technique that is highly effective, especially in this instance, where it eliminates the uncertainty of identifying the proper injection site in such patients. This technique for the interscalene brachial plexus block has proven to be simple, safe, reproducible, and highly successful. It is thus highly recommended for use by anesthesiologists and pain specialists alike.

\section{AUthOR AfFLLIATION:}

Edward Carden, MD

Clinical Professor

Department of Anesthesiology University of Southern California Keck School of Medicine, Los Angeles Southern California Academic Pain Management

13160 Mindanao Way, Suite 300

Marina Del Rey, CA 90292

E-mail: tedcarden@comcast.net

\section{Arti Ori, MBChB}

Attending Observership

Southern California Academic Pain

Management

13160 Mindanao Way, Suite 300

Marina Del Rey, CA 90292

E-mail: artiori1@yahoo.com

\section{REFERENCES}

1. Winnie AP. Interscalene brachial plexus block. Anesth Analg 1970; 49:455-466.

2. Manriquez RG, Pallares V. Continuous brachial plexus block for prolonged sympathectomy and control of pain. Anesth Analg 1978; 57:128-130.

3. Kirkpatrick AF, Bednarczyk LR, Hime GW. Bupivacaine blood levels during continuous interscalene block. Anesthesiology 1985; 62:65-67.

4. Brown A, Weiss R, Greenberg C, Flatow EL, Bigliano LU. Interscalene block for shoulder arthroscopy: comparison with general anesthesia. Arthroscopy 1993; 9:295-300.

5. D’Alessio J, Rosenblum M, Shea K, Freitas D. A retrospective comparison of interscalene block and general anesthesia for ambulatory surgery shoulder arthroscopy. Reg Anesth 1995; 20:62-68.

6. Urban MK, Urquhart B. Evaluation of brachial plexus anesthesia for upper extremity surgery. Reg Anesth 1994; 19:175-182.

7. Winnie AP. Plexus anesthesia: Perivascular techniques of brachial plexus block. W.B. Saunders, Philadelphia, 1983, pp 228234.

8. Rosenberg AD, Grande CM, Bernstein RL. Pain Management and regional anesthesia in trauma. Bailliere Tindall: 2000; pp 209-238.

9. Brown DL, Bridenbaugh LD. The upper extremity: The somatic block. In Cousins MJ, Bridenbaugh DO (eds). Neural blockade in clinical anesthesia and management of pain, third edition. Lippincott Williams \& Williams, Philadelphia, 1998, pp 345-371.

10. Digistim 2 Plus. Neuro Technology Inc., P.O. Box 291348, Kerrville, TX 78029.

11. 22-gauge insulated regional block needle with injection set. Kimberly Clark Corporation, Draper, UT 84020.

12. Silverstein WB, Saiyed MU, Brown AR. Interscalene block with a nerve stimulator: a deltoid motor response is a satisfactory endpoint for successful block. Reg Anesth Pain Med 2000; 25:356-359.

13. Dogru K, Duygulu F, Yildiz K, Kotanoghu MS, Modenoglu H, Boyaci A. Hemodynamic and blockade effects of high/low epinephrine doses during axillary plexus blockade with lidocaine $1.5 \%$ : A randomized, double-blinded study. Reg Anesth Pain Med 2003; 28:401-405.

14. Pihlajamaki KK, Lindberg RL. Bupivacaine with and without adrenaline in interscalene brachial plexus blockade. Studies in patients with rheumatoid arthritis. $\mathrm{Br} / \mathrm{An}$ aesth 1987; 59:1420-1424.

15. Tschirren B. Anesthetic complications: Etiology, prophylaxis, and treatment. Year Book Medical Publishers, Chicago, 1980.

16. Finucane BT (ed). Complications of regional anesthesia. Churchill Livingstone, New York, 1999, pp 271-291.

17. Raj PP (ed). Textbook of regional anesthesia. Churchill Livingstone, New York, 2002. 
18. Goldberg ME, Gregg C, Larijani GE. A comparison of three methods of axillary approach to brachial plexus blockade for upper extremity surgery. Anesthesiology 1987; 66:814-816.

19. Selander D, Edshage S, Wolff T. Paresthesiae or no paresthesiae? Nerve lesions after axillary blocks. Acta Anaesthesiol Scand 1979; 23:27-33.
20. Norris D, Klahsen A, Milne B. Delayed bilateral spinal anesthesia following interscalene brachial plexus block. Can J Anaesth 1996; 43:303-305.

21. Scammel SJ. Case report: Inadvertent epidural anaesthesia as a complication of interscalene brachial plexus block. Anaesth Intensive Care 1979; 7(1):56-57.

22. Urmey WF, McDonald M. Hemidiaphrag- matic paresis during interscalene brachial plexus block: effects on pulmonary function and chest wall mechanics. Anesth Analg 1992; 74:352-357.

23. Pauchet V, Sourdat P, Labat G. Regional Anesthesia, fourth edition. Doin, Paris, 1928, pp 222-225. 